\title{
MAXIMUM LIKELIHOOD BASED SPARSE AND DISTRIBUTED CONJOINT ANALYSIS
}

\author{
Efthymios Tsakonas *, Joakim Jaldén *, Nicholas D. Sidiropoulos ${ }^{\ddagger}$, Bjorn Ottersten *† \\ * ACCESS Linnaeus Centre, Royal Institute of Technology (KTH), Stockholm, Sweden \\ $¥$ Department of Electrical and Computer Engineering, University of Minnesota, Minneapolis \\ ${ }^{\dagger}$ Interdisciplinary Centre for Security, Reliability and Trust, University of Luxembourg, Luxembourg
}

\begin{abstract}
A new statistical model for choice-based conjoint analysis is proposed. The model uses auxiliary variables to account for outliers and to detect the salient features that influence decisions. Unlike recent classification-based approaches to choice-based conjoint analysis, a sparsity-aware maximum likelihood (ML) formulation is proposed to estimate the model parameters. The proposed approach is conceptually appealing, mathematically tractable, and is also wellsuited for distributed implementation. Its performance is tested and compared to the prior state-of-art using synthetic as well as real data coming from a conjoint choice experiment for coffee makers, with very promising results.
\end{abstract}

\section{INTRODUCTION}

In recent years we have witnessed a remarkable increase in the amount of data that is being collected regarding people's preferences about products, services, as well as other information sources. This increase has been largely stimulated by the growth of online retailing, social networking, personalized recommendation systems, and location-aware services. Given the substantial increase of preference data (choices, rankings, surveys, questionnaires) generated mainly through the web, there has been a growing interest in the area of preference modeling and analysis (PMA). As the name implies, the goal of PMA is to predict responses of individuals based on previously observed preference data.

Conjoint analysis (CA) has been a workhorse of PMA for over 30 years. CA has found exciting applications in marketing, industrial design, economics and beyond. The main assumption behind CA modeling is that responses are formed as noisy linear combinations of a product's features with weights given by the decision-maker's partworths. In reality, linearity is only an approximation [1]. Given observed preference data, CA techniques aim to estimate the underlying partworths from the available preference data. Partworths can be used to predict future preferences, but are also useful per se to the retailer, marketer, or product designer, e.g., for consumer sensitivity analysis.

Traditional methods for partworth estimation for choice-based CA models (where preferences are only expressed in the form of choices), range from logistic regression [2] and hierarchical Bayes methods [3, 4], to methods based on support vector machine (SVM) classifiers [5]. Following either deterministic or Bayesian formulations, these state-of-the-art techniques rely on suitably regularized

The research leading to these results has received funding from the European Research Council under the European Community's Seventh Framework Programme (FP7/2007-2013) / ERC grant agreement number 228044, and SSF grant ICA08-0046. loss functions to "optimally" trade-off model fit for the generalization capability of the solution beyond the training data. See [6] for a compact description of these approaches; more detailed comparisons can also be found in [5].

Although the benefits of CA have been widely appreciated, the tacit assumption underlying most of the existing techniques is that the data is gathered under controlled conditions, i.e., there are no outliers, and responses regress upon a modest number of features. However, in modern preference modeling systems such controlled conditions are often not possible. Therefore, solutions that are computationally efficient and offer robustness to gross errors are, if not necessary, at least highly desirable. Outlier-aware SVM classifiers for choice-based conjoint data are proposed in [5]. Mathematically, the approach in [5] requires solving an unconstrained optimization problem consisting of a loss function which is convex but not differentiable (known as the hinge loss function [7]) combined with a suitable regularizing function; in particular, the sum of squares of partworths. This regularization gives up some model fit but is important, as it improves the generalization capabilities of the classifier. Similarly to [5], the authors in [8] follow a SVM approach to choice-based CA, the main difference being that sparse outliers are modeled explicitly using auxiliary variables.

A different viewpoint is adopted in our paper, where a novel statistical choice-based CA model is proposed. The model includes both standard errors and auxiliary variables that explicitly model sparse outliers, and performs sparsity control on the partworths to identify the salient features that influence decisions. Departing from the approaches in $[5,8]$, we propose a ML estimator for the unknown model parameters, and incorporate sparsity information through $\ell_{1}$ norm penalties in the likelihood maximization problem. Unlike an SVM approach, the proposed ML metric is differentiable, which is advantageous in terms of mathematical tractability. We show how the approach can be extended to a distributed optimization framework and derive a simple decentralized algorithm based on the alternating direction method of multipliers (ADMM), a method which has shown great promise in the area of distributed optimization [7]. Due to differentiability, the individual nodes in the proposed distributed algorithm need only use the Newton's method [9] (or even simpler, gradient-based, methods) to cope with their individual optimizations. Finally, the validity of our model and the efficacy of the proposed sparsity-aware ML estimator is assessed using both simulated and real data from a conjoint choice experiment for coffee makers.

The rest of the paper is organized as follows. Section 2 describes the proposed model and main problem setup. The ML estimator that also accounts for outliers and partworth sparsity is derived in Section 3. Section 4 gives a distributed implementation of the proposed method based on ADMM. Numerical results are presented in Section 5 , and conclusions are drawn in Section 6. 


\section{CONTEXT AND MOTIVATION}

We start by describing the basic models in PMA. From now on, it is convenient to represent the quantities to be rated (and let us assume that these are products, for simplicity) using associated profiles, i.e., $p$-dimensional vectors whose elements correspond to the different features. Suppose there are $J$ such profiles $\left\{\mathbf{p}_{j}\right\}_{j=1}^{J}$, to be rated by a single individual. In PMA it is customary to assume that responses $\left\{r_{i}\right\}_{i=1}^{J}$ obey a linear regression model (see, e.g., [1])

$$
r_{i}=\mathbf{p}_{i}^{\mathrm{T}} \mathbf{w}+e_{i}
$$

where $\mathbf{w}$ is the vector of partworths associated with the individual and $e_{i}$ is a random variable modeling (usually small) random errors.

There are three different but related categories of models that link responses to preference measurements. In a full-profile rating model, the measurement is assumed to be directly the response $r_{i}$. Another category consists of the so-called metric-paired rating models, where the $\mathbf{p}_{i}$ in (1) is replaced by a difference $\mathbf{d}_{i} \triangleq \mathbf{p}_{i}^{(1)}-\mathbf{p}_{i}^{(2)}$ of a pair of profiles. Finally, we have also choice-based models, where in addition to using pairwise-differences of profiles in (1), the measurement is only the sign of $r_{i}$. In other words, in a choice-based CA model the individual is each time asked to indicate a preference between two profiles, but not the actual magnitude of this preference. Mathematically speaking, if we assume $N$ given profile differences $\left\{\mathbf{d}_{i}\right\}_{i=1}^{N}$ the classical choice-based CA model is

$$
y_{i}=\operatorname{sign}\left(\mathbf{d}_{i}^{\mathrm{T}} \mathbf{w}+e_{i}\right), \quad i=1, \cdots, N .
$$

An intuitive advantage of choice-based conjoint data models as compared to models based on rating scales is that choices are more realistic, resembling the real purchasing situation. Another advantage is that the problem of individual differences in interpreting rating scales is circumvented [10]. In this paper we deal exclusively with (2). We assume that the errors $\left\{e_{i}\right\}_{i=1}^{N}$ in (1) are drawn from an i.i.d. normal distribution $\mathcal{N}\left(0, \sigma^{2}\right)$ with known variance $\sigma^{2}$, and we aim to robustify the model based on two observations: The first is that responses themselves can be grossly inconsistent, owing to a number of different reasons. This implies that it is more realistic to assume that there can be gross errors in the response model in (1), in addition to the typically small errors $\left\{e_{i}\right\}_{i=1}^{N}$. However, it makes sense to assume that gross errors are sparse, since intuitively, an individual will not be regularly inconsistent. Second, the number of attributes $p$ can be very large-modern products have dozens of potentially relevant attributes and technical specifications-yet relatively few features will matter to any given individual, and even the 'typical' individual. This suggests that the unknown w will also be a sparse vector.

Hence, the model in (2) can be re-stated by (a) explicitly modeling the gross errors using a sparse vector $\mathbf{o} \in \mathbb{R}^{N \times 1}$ of variables $\left\{o_{i}\right\}_{i=1}^{N}$ and (b) utilizing the prior knowledge that $\mathbf{w}$ itself is a sparse vector. Therefore, a conceptually appealing version of (2) is

$$
y_{i}=\operatorname{sign}\left(\mathbf{d}_{i}^{\mathrm{T}} \mathbf{w}+e_{i}+o_{i}\right), \quad i=1, \cdots, N
$$

coupled with the a-priori knowledge that $\operatorname{card}(\mathbf{w}) \leq \kappa_{w}$ and card $(\mathbf{o}) \leq \kappa_{o}$. Here, the integers $\kappa_{w}, \kappa_{o}$ are assumed fixed and given, and the function card(.) stands for cardinality, i.e., it returns the number of non-zero elements of a vector.

\section{SPARSITY-AWARE ML ESTIMATOR}

Given the $N$ measurements as above and the aforementioned prior knowledge on $\mathbf{o}$ and $\mathbf{w}$, one may construct the ML estimator for the vector of partworths $\mathbf{w}$, as follows:
Let $\mathcal{I}_{+}$be the set of indices $\left\{i \mid y_{i}=1\right\}$, and similarly define $\mathcal{I}_{-}=\left\{i \mid y_{i}=-1\right\}$. Since noise samples $e_{i}$ are independent, the probability of a random partition of the observations to $\mathcal{I}_{+}$and $\mathcal{I}_{-}$ can be calculated explicitly to be $p_{y}(\mathbf{w}, \mathbf{o})=$

$$
\begin{aligned}
& =\prod_{i \in \mathcal{I}_{+}} \operatorname{Pr}\left[\mathbf{d}_{i}^{\mathrm{T}} \mathbf{w}+o_{i} \geq-e_{i}\right] \prod_{i \in \mathcal{I}_{-}} \operatorname{Pr}\left[\mathbf{d}_{i}^{\mathrm{T}} \mathbf{w}+o_{i} \leq-e_{i}\right] \\
& =\prod_{i \in \mathcal{I}_{+}} \Phi\left(\frac{\mathbf{d}_{i}^{\mathrm{T}} \mathbf{w}+o_{i}}{\sigma^{2}}\right) \prod_{i \in \mathcal{I}_{-}} \Phi\left(-\frac{\mathbf{d}_{i}^{\mathrm{T}} \mathbf{w}+o_{i}}{\sigma^{2}}\right)
\end{aligned}
$$

where $\Phi(u):=\frac{1}{\sqrt{2 \pi}} \int_{-\infty}^{u} e^{-t^{2} / 2} d t$ is the cumulative distribution function of the Gaussian density. The log-likelihood function can be written compactly as

$$
l(\mathbf{w}, \mathbf{o})=\log p_{y}(\mathbf{w}, \mathbf{o})=\sum_{i=1}^{N} \log \Phi\left(\frac{y_{i} \mathbf{d}_{i}^{\mathrm{T}} \mathbf{w}+y_{i} o_{i}}{\sigma^{2}}\right) .
$$

Therefore, the problem of finding the ML estimate of the vector $\mathbf{w}$ can be expressed as

$$
\begin{aligned}
& \underset{\mathbf{w}, \mathbf{o}}{\operatorname{maximize}} l(\mathbf{w}, \mathbf{o}) \\
& \text { subject to: } \operatorname{card}(\mathbf{w}) \leq \kappa_{w}, \quad \operatorname{card}(\mathbf{o}) \leq \kappa_{o} .
\end{aligned}
$$

It is well known that the objective $l(\mathbf{w}, \mathbf{o})$ in (5a) is concave (see e.g., [9, Ch. 3]) but the cardinality constraints on $\mathbf{w}$ and $\mathbf{o}$ are generally intractable. In order to obtain a tractable approximation, a common practice is to replace the cardinality constraints in $(5 \mathrm{~b})$ with convex $\ell_{1}$-norm constraints. This is motivated since the $\ell_{1}$-norm is the tightest convex relaxation of the cardinality function [9]. Such a replacement yields the convex program

$$
\begin{aligned}
& \underset{\mathbf{w}, \mathbf{o}}{\operatorname{maximize}} l(\mathbf{w}, \mathbf{o}) \\
& \text { subject to: }\|\mathbf{w}\|_{1} \leq \kappa_{w},\|\mathbf{o}\|_{1} \leq \kappa_{o}
\end{aligned}
$$

which can be solved efficiently, using, e.g., modern interior point methods [9]. Finally, a more compact way of expressing (6) is

$$
\underset{\mathbf{w}, \mathbf{o}}{\operatorname{minimize}} \phi(\mathbf{w}, \mathbf{o})=-l(\mathbf{w}, \mathbf{o})+\lambda_{w}\|\mathbf{w}\|_{1}+\lambda_{o}\|\mathbf{o}\|_{1}
$$

since (6) and (7) can be shown to be equivalent for a suitable choice of the regularization parameters $\lambda_{w}$ and $\lambda_{o}$. These control the tradeoff between $l(\mathbf{w}, \mathbf{o})$ and the number of non-zero elements of $\mathbf{w}$ and o respectively. In practice, the penalty parameters are tuned empirically: One starts from a suitable initial point $\left(\lambda_{w}^{i}, \lambda_{o}^{i}\right)$ and iterates until the desired sparsity / fit trade-off is achieved. Some assistance may be drawn from the following proposition.

Proposition 1. The point $\left(\mathbf{w}^{\star}, \mathbf{o}^{\star}\right)=\mathbf{0}$ is optimal for problem (7) if and only if $\lambda_{w} \geq\left\|\nabla_{w} l(\mathbf{0})\right\|_{\infty}$ and $\lambda_{o} \geq\left\|\nabla_{o} l(\mathbf{0})\right\|_{\infty}$, where $\nabla_{w} l(\mathbf{0})$ and $\nabla_{o} l(\mathbf{0})$ denote the gradients of $l(\mathbf{w}, \mathbf{O})$ with respect to $\mathbf{w}$ and $\mathbf{o}$ respectively, evaluated at $(\mathbf{w}, \mathbf{o})=\mathbf{0}$.

The proof follows directly from subdifferential calculus and is omitted for brevity. Therefore, for $\lambda_{w} \geq \lambda_{w}^{\max }=\left\|\nabla_{w} l(\mathbf{0})\right\|_{\infty}$ and $\lambda_{o} \geq \lambda_{o}^{\max }=\left\|\nabla_{o} l(\mathbf{0})\right\|_{\infty}$, the minimization in (7) yields the sparsest possible pair $(\mathbf{w}, \mathbf{o})$, the zero vector. A reasonable heuristic approach to tune the parameters is to initialize by choosing $\lambda_{w}=$ $\lambda_{w}^{\max } / 2$ and $\lambda_{o}=\lambda_{o}^{\max } / 2$, and adjust to achieve the desired sparsity / fit trade-off. Devising systematic methods on how to choose the 
penalty parameters is an important topic on its own which deserves further investigation.

Finally, in closing the section, we remark that the regularized ML estimator proposed in (7) may be viewed as a maximum aposteriori probability (MAP) estimator of $\mathbf{w}$ and $\mathbf{o}$, which is very common in statistics [11]. The condition under which our approach becomes equivalent to the MAP estimator is when it is assumed that both $\mathbf{w}$ and $\mathbf{o}$ are random with a Laplacian prior.

\section{DISTRIBUTED CHOICE-BASED CA}

Problem (7) is a convex optimization problem that can be solved using a variety of algorithms, including interior point methods that feature low-order polynomial worst-case complexity. This is encouraging, of course, but still far from practical applications. Online retail and social networking sites, for example, generate huge volumes of expressed preference data, which far outweigh the ability of a single modern computer to analyze them in real-time. Also, because datasets in such applications are usually very large, they are often stored (or collected) in a distributed fashion. It is therefore of interest to develop distributed solution methods to process huge datasets in real-time, possibly using multiple processors. Confidentiality issues are another motivation for distributed solution methods.

For solving (7) in a distributed manner, we propose an algorithm based on the ADMM. The basic ADMM algorithm was developed in the 1970's and has recently become a very popular choice for solving large-scale problems. The name derives from the fact that the algorithm alternates between optimizing different variables in the augmented Lagrangian function. It is closely related to other algorithms, such as Douglas-Rachford splitting, the split Bregman method, and the method of multipliers. See [7] and references therein for a recent review on ADMM.

The objective of (7) is additive with respect to the observed data, and there is a single global variable $\boldsymbol{\xi} \triangleq\left[\mathbf{w}^{\mathrm{T}} \mathbf{o}^{\mathrm{T}}\right]^{\mathrm{T}} \in \mathbb{R}^{p+N}$. If we assume that the observed data are partitioned into $K$ blocks $\left\{N_{i}\right\}_{i=1}^{K}$, then the goal is to split the objective of (7) into $K$ terms, and give each term to be handled by its individual processing unit (such as a thread or processor). Let us denote as $l_{i}(\mathbf{w}, \mathbf{o})$ the function summing the cost of all examples in block $N_{i}$, i.e.,

$$
l_{i}(\mathbf{w}, \mathbf{o}) \triangleq \sum_{j \in N_{i}} \log \Phi\left(\frac{y_{j} \mathbf{d}_{j}^{\mathrm{T}} \mathbf{w}+y_{j} o_{j}}{\sigma^{2}}\right) .
$$

Introducing the local variables $\boldsymbol{\xi}_{\boldsymbol{i}} \triangleq\left[\mathbf{w}_{i}^{\mathrm{T}} \mathbf{o}_{i}^{\mathrm{T}}\right]^{\mathrm{T}} \in \mathbb{R}^{p+N}$ and the global variable $\mathbf{z} \triangleq\left[\begin{array}{ll}\mathbf{z}_{1}^{\mathrm{T}} & \mathbf{z}_{2}^{\mathrm{T}}\end{array}\right]^{\mathrm{T}} \in \mathbb{R}^{p+N}$, one can equivalently write problem (7) in its consensus form [7]

$$
\begin{aligned}
& \underset{\left\{\boldsymbol{\xi}_{\boldsymbol{i}}\right\}_{i=1}^{K}, \mathbf{z}}{\operatorname{minimize}}-\sum_{i=1}^{K} l_{i}\left(\boldsymbol{\xi}_{\boldsymbol{i}}\right)+h(\mathbf{z}) \\
& \text { subject to: } \boldsymbol{\xi}_{\boldsymbol{i}}-\mathbf{z}=\mathbf{0}, \quad i=1, \cdots, K
\end{aligned}
$$

where the regularization function $h: \mathbb{R}^{p+N} \rightarrow \mathbb{R}$ is defined as $h(\mathbf{z}) \triangleq \lambda_{w}\left\|\mathbf{z}_{1}\right\|_{1}+\lambda_{o}\left\|\mathbf{z}_{2}\right\|_{1}$. Problem (9) is called the global consensus problem, owing to the consensus constraint [in (9b)] which enforces all the local variables to be equal. This can be solved by applying the generic global variable consensus ADMM algorithm described in [7, Ch. 7]. The full derivation of the distributed algorithm will be presented in an extended version of this work; here we only present and explain the basic steps of the distributed algorithm. Upon defining the dual variables $\mathbf{u}_{i} \in \mathbb{R}^{p+N}$ and a fixed parameter $\rho>0$ (often called the penalty parameter), the algorithm consists of the following three main updates:

$$
\begin{aligned}
\boldsymbol{\xi}^{k+1} & :=\underset{\boldsymbol{\xi}_{i}}{\arg \min }-l_{i}\left(\boldsymbol{\xi}_{\boldsymbol{i}}\right)+(\rho / 2)\left\|\boldsymbol{\xi}_{\boldsymbol{i}}-\mathbf{z}^{k}+\mathbf{u}_{i}^{k}\right\|_{2}^{2} \\
\mathbf{z}^{k+1} & :=\underset{\mathbf{z}}{\arg \min } h(\mathbf{z})+(K \rho / 2)\left\|\mathbf{z}-\overline{\boldsymbol{\xi}}^{k+1}-\overline{\mathbf{u}}^{k}\right\|_{2}^{2} \\
\mathbf{u}_{i}^{k+1} & :=\mathbf{u}_{i}^{k}+\boldsymbol{\xi}_{\boldsymbol{i}}{ }^{k+1}-\mathbf{z}^{k+1} .
\end{aligned}
$$

The step in (10a) can be carried out in parallel for each data block. As opposed to an SVM distributed implementation [7], note here that each $l_{i}\left(\boldsymbol{\xi}_{\boldsymbol{i}}\right)$ is a differentiable function. Therefore, the minimization in this step can be carried out efficiently using gradientor Hessian-based methods (e.g., Newton's method). To further increase efficiency warm starts can be used, i.e., initializing from the previously computed data point. The second step requires gathering the variables to form the averages, which are denoted as $\bar{\xi}^{k+1}$ and $\overline{\mathbf{u}}^{k}$. Observe that the regularization function $h$ is fully separable in the global variable $\mathbf{z}$, therefore the minimization in (10b) can be carried out component-wise.

Following a random initialization, the above iterations are guaranteed to converge to an optimal point for (9) as $k \rightarrow \infty$. In practice, although ADMM can be very slow to converge to high accuracy, it usually converges to modest accuracy within a few tens of iterations [7]. Thankfully, our simulation examples indicate that modest accuracy is sufficient in this context, motivating the practical use of this algorithm.

Note that the end-result is an algorithm which is not only distributed, but also decentralized: A node does not need access to the individual data of another-only the consensus variable $\mathbf{z}$ is needed to be shared for convergence. Even for modestly sized datasets, such decentralized solutions might be preferable from centralized ones due to many reasons (for example, due to the privacy requirements).

\section{NUMERICAL RESULTS}

In this section we compare our distributed implementation in (10) against a particular SVM variant inspired by [5]. We use both synthetic and real data coming from a conjoint choice experiment for coffee makers.

\subsection{Synthetic data}

The metric chosen for the comparison is the Root Mean Squared Error (RMSE) between the estimated and "true" partworths, after both have been normalized in $\left(\ell_{2}\right.$-norm) magnitude for comparability. The same metric was also adopted in $[5,8]$. The RMSE of the two methods was estimated using $\mathrm{MC}=50$ Monte Carlo trials. For each trial, product profiles were generated as i.i.d Gaussian vectors, each comprising $p=20$ attributes/features. For each trial we constructed $N=500$ choice questions, by constructing vector differences randomly among the generated profiles. We considered two different settings: $(i)$ one where all $N=500$ choice questions were used for the purpose of estimation, and $(i i)$ a reduced-size (questionnaire) setting, where 50 choice questions were randomly drawn from this complete set of 500. Choice data were generated according to model (3). We experimented using two different outlier percentages in the responses, $4 \%$ and $10 \%$ (outliers correspond to sign change in $y_{i}$ ). The unknown partworth vector was assumed sparse ( $\mathrm{NZ}=3$ non-zero entries) i.i.d. Gaussian. To be fair in this comparison, prior knowledge about the partworth vector sparsity was added 
in the SVM proposed in [5] as well. This was done by adding an extra $\ell_{1}$-norm penalty for $\mathbf{w}$ to the objective of [5, Problem (3)]. The resulting SVM variant (abbreviated here as DR-SVM) was first proposed in [12]. For our distributed ML estimator we assumed $K=5$ clusters of data of equal size, and a penalty parameter $\rho=1$. The same stopping criterion as in [7, Ch. 3] was used to terminate the iterations. Choosing the regularization parameters for both algorithms requires extra care, since cross validation can be inefficient when outliers are present [8]. In this simple experiment we assumed that the degree of sparsity in both $\mathbf{w}$ and $\mathbf{o}$ is known a-priori. The choice of the parameters was done heuristically, searching inside the box $\left[0,5 \lambda_{w}^{\max }\right] \times\left[0,5 \lambda_{o}^{\max }\right]$, using Proposition 1 both for initialization and also to exclude the points that would yield the all-zero vector as a solution.

The results of the RMSE comparison are reported in Table 1. The table also includes the RMSE given by the exact SVM proposed in [5] (without the additional $\ell_{1}$-norm regularizer on $\mathbf{w}$ ) for completeness. Note that although the ML estimator is slightly inferior (but nonetheless competitive) to the DR-SVM for the reduced-sized questionnaire with a small percentage of outliers, it clearly outperforms the DR-SVM as the number of samples becomes large enough. Observe also that the exact SVM from [5] is always inferior than the other two methods, but this is expected of course, as [5] was not designed to account for partworth sparsity, and this is the reason we have included DR-SVM in the comparison.

\begin{tabular}{|c|c|c|c|c||}
\hline Outliers & Questions & SVM[5] & DR-SVM & Proposed (10) \\
\hline \hline $4 \%$ & 50 & 0.6976 & $\mathbf{0 . 1 7 8 0}$ & 0.1830 \\
\hline $4 \%$ & 500 & 0.0259 & 0.0112 & $\mathbf{0 . 0 0 6 2}$ \\
\hline $10 \%$ & 50 & 0.7284 & 0.1817 & $\mathbf{0 . 1 5 2 3}$ \\
\hline $10 \%$ & 500 & 0.0328 & 0.0161 & $\mathbf{0 . 0 0 9 7}$ \\
\hline \hline
\end{tabular}

Table 1. RMSE comparison of the three methods: SVM from [5], DR-SVM, and the proposed method (10). The method that yields lower RMSE is marked with bold.

\subsection{Real data}

Although the above results appear promising, the synthetic data were generated according to the model in (3), which is clearly in favor of our ML estimator. Here, in a similar comparison, we use real data that may violate our assumptions. We briefly describe the general setup; all details can be found in [10, Ch. 13.6].

Hypothetical coffee makers were defined using the following five attributes:

- [Brand] brand-name (Phillips, Braun, Moulinex)

- [Capacity] number of cups $(6,10,15)$

- [Price] price in Dutch Guilders f: $(39,69,99)$

- [Thermos] presence of a thermos flask (yes, no)

- [Filter] presence of a special filter (yes, no)

A total of sixteen profiles were constructed from combinations of the levels of these attributes using an incomplete design [10]. These sixteen profiles are represented mathematically as vectors in $\mathbb{R}^{7}$ (with three binary entries describing the brand of the product). In the choice experiment, respondents were asked to make choices out of sets of three profiles, each set containing the same base alternative [10]. Therefore, each choice expresses two strict preferences between different coffee makers. In total, 185 respondents were recruited in the experiment and each one provided data for 16 choices. Links for the actual dataset used in this part can be found in [10].
We tested our ML estimator in (7) and the SVM partworth estimator from [5]. Our metric in this case was the predictive performance, or the "hit-rate" of each method, which we assessed by reserving the last out of the 16 choices and testing how often the estimated utility functions predict the correct winning product. This time no prior knowledge about sparsity was incorporated in the SVM approach - we test the exact SVM proposed in [5]. The regularization parameter for the SVM was tuned using the leave-one-out error approach described in [5]. For the ML estimator, the parameters were adjusted by assuming $i$ ) outlier frequency of $1 / 16$ and $(i i)$ a sparse $\mathbf{w}$ having only NZ $=2$ non-zeros. The RMSE results are at least encouraging: The ML estimator predicted the correct choice in 174 out of 185 cases (94\%), while the SVM predicted the winning product correctly in 169 cases $(91.3 \%)$.

\section{CONCLUSION}

The paper proposes a new method for choice-based CA. The main contribution is a statistical choice model which offers robustness against sparse outliers and performs parsimonious feature selection. The associated ML estimator leads to a formulation which can efficiently handle large scale datasets through a simple distributed implementation, which seems to perform very well in practice.

\section{REFERENCES}

[1] A. Gustafsson, A. Herrmann, F. Huber, Conjoint Measurement: Methods and Applications, Springer-Verlag, Berlin, 2007.

[2] J. Louviere, D. Hensher, J. Swait, Stated Choice Methods: Analysis and Applications, Cambridge University Press, UK, 2000.

[3] W. DeSarbo, A. Ansari, "Representing heterogeneity in consumer response models," Marketing letters, 8(3) 335-348.

[4] P. Lenk, W. DeSarbo, P. Green, M. Young “ Hierarchical Bayes Conjoint Analysis: Recovery of Partworth Heterogeneity from Reduced Experimental Designs", Marketing Science, vol. 15, no. 2, pp. 173-191, 1996.

[5] T. Evgeniou, C. Boussios, and G. Zacharia, "Generalized robust conjoint estimation," Marketing Science, vol. 24, no. 3, pp. 415$429,2005$.

[6] O. Chapelle and Z. Harchaoui, "A machine learning approach to conjoint analysis," in Advances in Neural Information Processing Systems, volume 17, pages 257-264. MIT Press, 2005.

[7] S. Boyd, N. Parikh, E. Chu, B. Peleato and J. Eckstein, "Distributed Optimization and Statistical Learning via the Alternating Direction Method of Multipliers," Foundations and Trends in Machine Learning, 2010, pp. 1-122.

[8] G. Mateos and G. B. Giannakis, "Robust Conjoint Analysis by Controlling Outlier Sparsity," in Proc. of European Signal Processing Conference, Barcelona, Spain, Aug. 29- Sep. 2, 2011.

[9] S. Boyd, and L. Vandenberghe, Convex Optimization, Cambridge University Press, 2004.

[10] Anders Skrondal and Sophia Rabe-Hesketh, Generalized Latent Variable Modeling: Multilevel, Longitudinal, and Structural Equation Models, Chapman \& Hall/CRC: Boca Raton, FL.

[11] S.M. Kay, Fundamentals of Statistical Signal Processing: Estimation Theory, Prentice-Hall, Englewood Cliffs, NJ, 1993.

[12] L. Wang, J. Zhu and H. Zou, "The doubly regularized support vector machine”, in Statistica Sinica, 16: 589-615, 2006. 